Check for updates

Cite this: RSC Adv., 2018, 8, 26987

\title{
Rhodamine B degradation by nanosized zeolitic imidazolate framework-8 (ZIF-8) $\uparrow$
}

\author{
Michael Chin, Cecilia Cisneros, Stephanie M. Araiza, Kevin M. Vargas, Kristi M. Ishihara \\ and Fangyuan Tian (D) *
}

Adsorption and photodegradation of rhodamine B by ZIF-8 nanocrystals were studied using spectroscopic techniques coupled with density functional theory (DFT) cluster calculations. A fast adsorption rate was observed in the dark, but upon exposure to visible light or UV irradiation the adsorption rate noticeably increased. Although several studies previously reported this phenomenon involving bulk ZIF-8 powder, this is the first mechanistic study to our knowledge that demonstrates adsorption and degradation of rhodamine $\mathrm{B}$ by nanosized ZIF- 8 under various light conditions. The combined study of $\mathrm{N}_{2}$ sorption pore analysis and surface-sensitive X-ray photoelectron spectroscopy (XPS) confirmed the surface adsorption was mainly due to the open metal sites and surface groups of nanoporous ZIF-8. The fluorescence studies suggested ZIF-8 nanocrystals were able to generate hydroxyl radicals in water but only under UV illumination. The work presented here provides an insight into understanding nanoscale metal-organic frameworks (MOFs) in the removal of organic molecules from wastewater.

Received 22nd April 2018

Accepted 19th July 2018

DOI: $10.1039 / \mathrm{c} 8 \mathrm{ra03459a}$

rsc.li/rsc-advances

bonding, and $\pi-\pi$ stacking. ${ }^{10-12}$ Compared to conventional

\section{Introduction}

Large amounts of organic dyes are consumed in various industrial applications involving textiles, paints and coatings, paper production, and plastic processing. Dye-containing wastewater is hazardous to the environment and can cause adverse health effects. ${ }^{1}$ It is important to effectively remove the threat of organic dyes from water. Typical water remediation treatments, such as nanofiltration and reverse osmosis, require excess power consumption and high-cost maintenance. ${ }^{2,3}$ Physical adsorption by immersing solid adsorbents in organic dye-containing wastewater is a low-cost alternative with high efficiency and efficacy. ${ }^{4}$

The focus of this study is to explore the use of a type of nanosized metal-organic framework (MOF) as a physical adsorbent for organic dye removal. MOFs are a type of hybrid material consisting of metal clusters connected by organic linkers forming a three-dimensional porous network. ${ }^{5}$ The high surface area, structural flexibility, and relatively good stability of certain MOFs have shown promise in water remediation and purification. So far, significant and inspiring studies have been performed in applying MOFs, such as MIL-101, UiO-66, and ZIF-8, for the removal of arsenic, sulfur-containing contaminants, heavy metals, and organic molecules in water. ${ }^{6-10}$ Some of these MOF materials are proven to be effective organic adsorbents based on external interactions, including electrostatic forces, hydrogen

Department of Chemistry and Biochemistry, California State University Long Beach, Long Beach, CA, 90840, USA. E-mail: fangyuan.tian@csulb.edu

$\dagger$ Electronic supplementary information (ESI) available: Additional TEM images, XPS, fluorescence, UV, gas isotherm, and XRD data. See DOI: 10.1039/c8ra03459a sorbents, such as zeolites, polymeric resins, and porous carbon, MOFs have shown significant advantages in organic dye degradation: first, the organic building block constructed MOFs are available for chemical modification, allowing rationale postsynthetic modification to introduce additional functional groups targeting removal of different dyes. ${ }^{13}$ Second, variable surface groups and large porosity range makes MOFs suitable for adsorbing organic pollutants of different sizes on the outer surfaces and within the pores. ${ }^{14}$ Third, the flexible structural nature of MOFs enables tuning of photocatalytic properties of MOFs by introducing variable active sites for photodegradation of dyes. Moreover, with more MOFs becoming commercially available, the cost of MOFs has dramatically decreased.

Zeolitic imidazolate framework-8 (ZIF-8), one of the most well-studied MOFs, exhibits excellent performance in degradation of organic dyes, such as methylene blue, triiodide, and rhodamine 6G., ${ }^{75,16}$ ZIF-8 is composed of tetrahedrally coordinated zinc ions connected with 2-methylimidazole (2-mIm), resulting in a sodalite (sod) zeolite structure. Bulk ZIF-8 crystals demonstrate a high specific surface area $\left(\sim 2100 \mathrm{~cm}^{2} \mathrm{~g}^{-1}\right)$ and porosity, making it attractive for a wide range of applications, such as gas sensing, separation and storage, catalysis, and membrane fabrications. ${ }^{17,18} \mathrm{ZIF}-8$ is chemically inert in aqueous solution with a $\mathrm{pH}$ value greater than $7 .{ }^{19}$ Furthermore, the size of ZIF-8 crystals can be tuned in a range of nano- to microscales. For example, Cravillon et al. and co-workers synthesized nanoscale ZIF-8 with an average diameter of $45 \mathrm{~nm}$ in methanol at room temperature. ${ }^{20}$ Recently, Fan et al. and co-workers prepared ZIF-8 nanocrystals with high surface area in aqueous 
condition. ${ }^{16}$ In their work, they noticed nanosized ZIF-8 is more favorable for the removal of organic dyes. Compared to its bulk material (in size of micrometers), nanoscale ZIF-8 exhibits higher external surface area with more surface groups, which have particular importance for adsorption, catalysis, and surface reactions. ZIF-8 has been experimentally confirmed to have a Zn-rich surface terminated with multiple functional groups, such as hydroxide, carbonates, and amines, in addition to methylimidazole groups. ${ }^{21}$ Very little research focusing on the role of surface groups concerning the study of organic dye@MOFs has been performed to date and such studies could advance the field significantly.

In this study, we explored the application of nanosized ZIF-8 as an adsorbent for dye removal from water. Rhodamine $\mathrm{B}$ (RhB) was selected as a model dye since it is a commonly used organic dye in many chemical and biological techniques. Additionally, $\mathrm{RhB}$ is detrimental to the environment with evidence of long lasting effects to aquatic life. ${ }^{22}$ Despite being known as a carcinogenic compound, it is used illegally as a food dye in many countries. ${ }^{23}$ Moreover, $\mathrm{RhB}$ is stable in water in a wide $\mathrm{pH}$ range and not biodegradable; therefore, it should be fully removed or decomposed before releasing RhB-containing wastewater to the environment. Although Fan et al. have compared nano-sized ZIF-8 and its bulk crystals for removal of $\mathrm{RhB},{ }^{\mathbf{1 6}}$ the mechanisms are still unclear. Our main interest in this study is to synthesize uniform ZIF-8 nanocrystals and apply it in organic dye removal, as well as to understand the role of surface groups on ZIF-8 nanocrystals during the organic adsorption process.

Here, we demonstrated nanosized ZIF-8 can be used for removing RhB in water under dark, visible light, and UV irradiation conditions. We employed $\mathrm{N}_{2}$ sorption and surface sensitive X-ray photoelectron spectroscopy (XPS) measurements in our studies of RhB adsorption on ZIF-8 nanocrystals since these techniques together provide a complete understanding of surface area and adsorption phenomena. A comparison of different behaviors of ZIF-8 nanocrystals toward RhB adsorption in dark/light conditions allowed us to predict various adsorption/degradation mechanisms. The experimental studies coupled with the density functional theory (DFT) cluster calculations helped us understand the interactions between $\mathrm{RhB}$ and the surface groups/defects of ZIF-8.

\section{Materials and methods}

\subsection{Chemicals}

All chemicals were reagent grade or better, used as received, and included zinc nitrate hexahydrate (Fisher Chemical, ACS certified), 2-methylimidazole (Acros, 99\%), rhodamine B (Acros, 98+\%); terephthalic acid (Acros, 99+\%), sodium hydroxide (Alfa Aesar, $2.0 \mathrm{~N}$ ), methanol (Fisher, ACS grade), and ethanol (Koptec, 190 proof pure).

\subsection{Synthesis of ZIF-8 nanoparticles}

2-Methylimidazole ( $\mathrm{mIm}$ ) (3.244 g) and zinc nitrate hexahydrate $\left(\mathrm{Zn}\left(\mathrm{NO}_{3}\right)_{2} \cdot 6 \mathrm{H}_{2} \mathrm{O}\right)(1.466 \mathrm{~g})$ were dissolved in separate portions of $100 \mathrm{~mL}$ methanol. The $\mathrm{Zn}\left(\mathrm{NO}_{3}\right)_{2} \cdot 6 \mathrm{H}_{2} \mathrm{O}$ solution was added to the $\mathrm{mIm}$ solution and the reaction was stirred rapidly for 1 hour. The resulting ZIF-8 nanoparticles were collected by centrifugation at $7500 \mathrm{rpm}$ for $30 \mathrm{~min}$ (Eppendorf, model 5430). The obtained ZIF-8 nanoparticles were redispersed in ethanol and centrifuged two more times before drying in a gravity oven overnight at $100{ }^{\circ} \mathrm{C}$.

\subsection{Characterization techniques}

2.3.1 $\mathbf{N}_{2}$ Sorption Studies. $\mathrm{N}_{2}$ sorption studies of nanoscale ZIF-8 before and after adsorbing RhB were carried out on a Micromeritics ASAP 2020PLUS surface area and pore size analyzer. Prior to measurements, samples were degassed through a multi-step heating process $\left(80{ }^{\circ} \mathrm{C}, 100{ }^{\circ} \mathrm{C}\right.$, and $\left.150{ }^{\circ} \mathrm{C}\right)$ under vacuum for over 12 hours to remove air trapped inside the pores of ZIF-8. The $\mathrm{N}_{2}$ sorption experiments were conducted at $77 \mathrm{~K}$ with approximately $0.1 \mathrm{~g}$ of powder samples.

2.3.2 Infrared (IR) spectroscopy. Infrared (IR) spectra were collected using an attenuated total reflectance infrared (ATR-IR) spectrometer (Bruker Instruments, Alpha I Platinum) in the range of $4000-400 \mathrm{~cm}^{-1}$ at a resolution of $4 \mathrm{~cm}^{-1}$ and a total of 128 scans per spectrum. The open air was used as background.

2.3.3 Powder X-ray diffraction (PXRD). Powder X-ray diffraction (PXRD) data was collected using a PANalytical X'pert Pro MPD diffractometer equipped with a linear X'Celerator detector and a $\mathrm{Cu} \mathrm{K} \alpha_{1}$ radiation source. Diffraction data was collected at room temperature in the range of 5-30 degrees with a $\sim 0.008$ step size.

2.3.4 Transmission Electron Microscopy (TEM). Samples of ZIF-8 nanoparticles diluted in ethanol were loaded onto 200 mesh carbon-coated copper grids with Formvar film (Ted Pella, Inc.) and allowed to air dry before imaging. TEM images were obtained using a JEOL 1200 EX II electron microscope operating at $100 \mathrm{kV}$. Particle size distribution analysis was conducted using ImageJ software.

2.3.5 X-ray Photoelectron Spectroscopy (XPS). X-ray photoelectron spectra were collected on an AXIS Supra (Kratos Analytical) with an Al monochromatic anode at energy of $1486.6 \mathrm{eV}$. The survey spectra were collected over the energy range of $0-1400 \mathrm{eV}$. The high-resolution spectra for each element ( $\mathrm{Zn} 2 \mathrm{p}, \mathrm{O} 1 \mathrm{~s}, \mathrm{~N}$ 1s, and C 1s) were collected over a range of $20 \mathrm{eV}$ at $0.1 \mathrm{eV}$ per step with a pass energy of $50 \mathrm{eV}$. Data analysis was performed using CasaXPS software (Casa Software, Ltd.). All peak positions were calibrated based on the binding energy of the adventitious C $1 \mathrm{~s}$ at $284.6 \mathrm{eV}$.

\subsection{Studies of adsorption kinetics}

The synthesized ZIF-8 nanoparticles were completely dried before mixing with RhB solution. $1 \mathrm{~L}$ RhB stock solution $(5.22 \times$ $10^{-4} \mathrm{M}$ ) was prepared then diluted to the desired concentrations with deionized water (18 $\mathrm{M} \Omega \mathrm{cm}$, Milli-Q water system, Millipore). A UV-Vis spectrometer (Shimadzu, UV-1800) was used to monitor the concentrations of RhB by measuring the absorbance at $554 \mathrm{~nm}\left(\lambda_{\max }\right)$. The UV-Vis standard curve was created based on a series of RhB solution in the concentration range of 1-10 ppm. For a typical kinetics measurement, $40 \mathrm{mg}$ 
of ZIF-8 was dispersed in $40 \mathrm{~mL}$ of $0.011 \mathrm{mM}$ RhB solution and stirred under dark, ambient light, and UV irradiation conditions (Spectronics Corp, SB-100PC, $365 \mathrm{~nm}$ ) at room temperature. UV-Vis absorbance was measured by extracting $1 \mathrm{~mL}$ of the sample using a $0.45 \mu \mathrm{m}$ syringe filter in the following time intervals: every minute for the first $10 \mathrm{~min}$, and every $10 \mathrm{~min}$ thereafter until a total stirring time of 60 min was reached. The absorbance measurements were performed in triplicate and the average absorbance was reported. The adsorption kinetics were evaluated using pseudo-first-order and pseudo-second-order models, respectively. If a linear relationship is observed, it confirms the adsorption follows the corresponding kinetics model.

\subsection{Studies of adsorption mechanisms}

The adsorption mechanisms were studied experimentally and theoretically. The surface interactions were evaluated by monitoring the $\mathrm{N}$ 1s and $\mathrm{Zn} 2 \mathrm{p}$ binding energies using XPS. Photocatalytic effect was studied by measuring ${ }^{\circ} \mathrm{OH}$ radical levels in ZIF-8/NaOH solution using terephthalic acid as a probe ( $40 \mathrm{mg}$ of ZIF-8 in $40 \mathrm{~mL}$ of $0.5 \mathrm{mM} \mathrm{NaOH}$ solution with $2 \mathrm{mM}$ of terephthalic acid). Terephthalic acid binds to ${ }^{\circ} \mathrm{OH}$ radicals and forms 2-hydroxyterephthalic acid which emits fluorescence at $430 \mathrm{~nm}$. The concentration of 2-hydroxyterephthalic acid was monitored by a fluorimeter (Shimadzu, RF-5301PC).

The RhB@ZIF-8 adsorption mechanisms were also studied by cluster calculations based on the density function theory (DFT). ZIF-8 was represented by a basic $\mathrm{Zn}$-imidazole cluster which has been confirmed as accurate as a periodic model. ${ }^{24}$ The molecular optimization and full natural bonding orbitals (NBO) were performed with the Gaussian 09 suite of programs using the B3LYP approximation to the exchange-correlated functional $^{25-27}$ with LANL2DZ effective core potential and valence basis set. ${ }^{\mathbf{2 8}, 29}$ The calculated nitrogen core-level energies were compared to experimental $\mathrm{N} 1 \mathrm{~s}$ binding energies by using a conversion factor of $8.50 \mathrm{eV}$ in water solvated conditions. ${ }^{30}$

\section{Results and discussion}

\subsection{Materials characterization}

The crystal structure of the nanosized ZIF- 8 was confirmed by PXRD (Fig. 1a) to be in excellent agreement with the simulated diffraction diagram and the previously reported patterns of bulk ZIF-8 powder. ${ }^{31,32}$ The chemical composition of ZIF-8 nanoparticles was examined by ATR-IR (Fig. 1b), and the observed IR absorption frequencies corresponding to functional groups are summarized in Table $\mathrm{S} 1 \uparrow$ and found to be consistent with those previously reported in the literature. ${ }^{33,34}$ We also investigated the morphology of the synthesized ZIF-8 nanoparticles. Fig. 1c shows a TEM image of freshly prepared ZIF-8, indicating uniform nanocrystals with an average diameter of $88.8 \pm$ $12.8 \mathrm{~nm}$. The histogram shown in Fig. 1d was calculated based on the average Feret diameter. More histogram fitting details can be found in the ESI (Fig. S1 $\dagger$ ).

The gas sorption behaviors and surface area of nanosized ZIF-8 were determined by the $\mathrm{N}_{2}$ sorption isotherms at $77 \mathrm{~K}$.
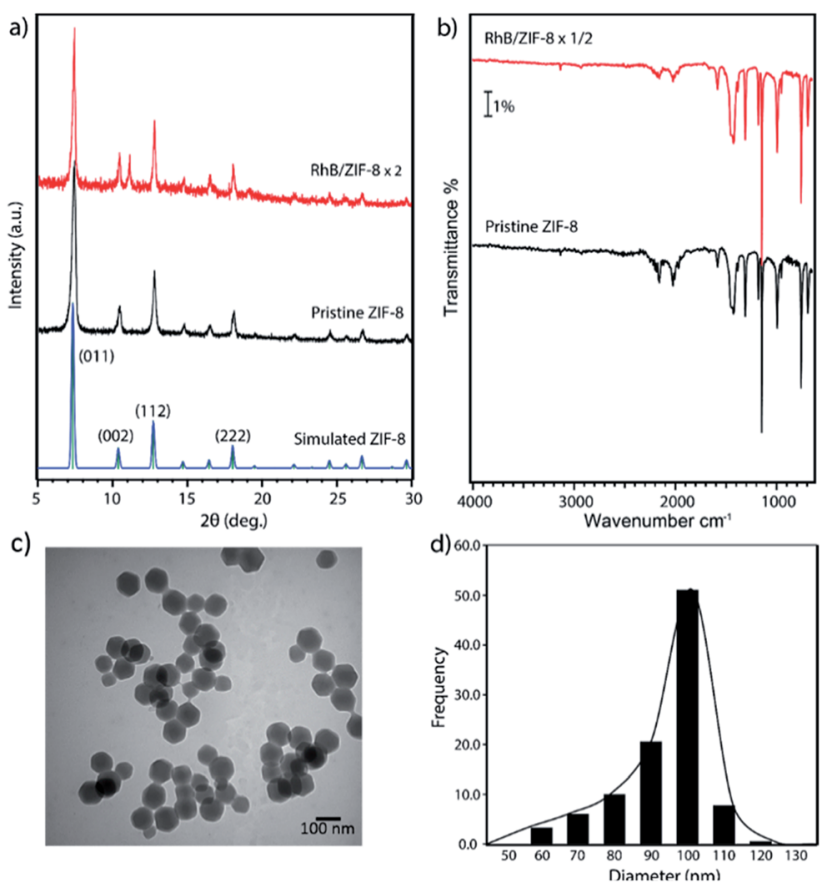

Fig. 1 (a) PXRD patterns of ZIF-8 nanoparticles before and after RhB adsorption compared with the simulated diagram based on the bulk ZIF- 8 crystal structure; (b) ATR-IR spectra of ZIF- 8 nanoparticles before and after RhB adsorption; (c) a TEM image of as-synthesized ZIF-8 nanoparticles; and (d) the ZIF-8 particle size distribution (adsorption condition: RhB, 5 ppm, under ambient light).

Fig. 2 shows the $\mathrm{N}_{2}$ adsorption and desorption isotherms of ZIF8 nanocrystals, confirming that all the pores of ZIF-8 were open after thermal activation. The rapid growth of $\mathrm{N}_{2}$ uptake amount at low pressure indicated the adsorption in microporous solids. Unlike bulk ZIF-8 with a typical type I isotherm, an H1 type hysteresis was observed in the high pressure region in the sorption isotherms, caused by the capillary condensation of $\mathrm{N}_{2}$ molecules adsorbed into the intraparticulate sites. ${ }^{35}$ Our previous studies also confirmed that gas molecules can be trapped among ZIF-8 nanoparticles when the adsorbate

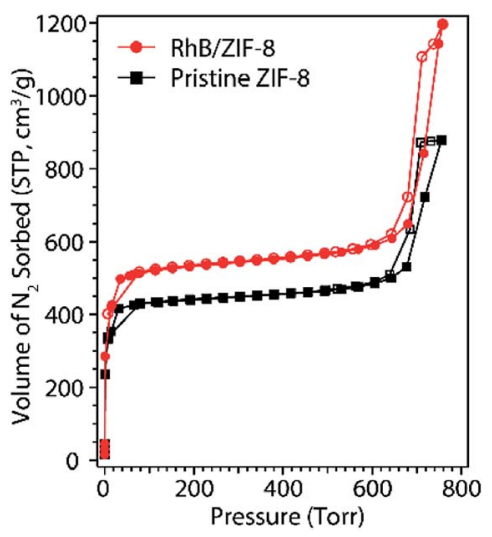

Fig. $2 \mathrm{~N}_{2}$ sorption isotherms of ZIF-8 nanoparticles at $77 \mathrm{~K}$ before and after RhB adsorption ( 5 ppm under ambient light). Solid symbols (square and circle) correspond to adsorption plots and open symbols correspond to desorption plots. 
concentration is higher than a monolayer coverage. ${ }^{36}$ The H1 type hysteresis loop is commonly observed in gas sorption isotherms of nanoparticles with uniform shape and facile pore connectivity, ${ }^{35,37,38}$ which agrees with our TEM observation of the ordered ZIF-8 nanoparticles. The gravimetric BrunauerEmmett-Teller (BET) surface area of the synthesized ZIF-8 was found to be $1698 \mathrm{~m}^{2} \mathrm{~g}^{-1}$, as consistent with those previously reported for nanoscale ZIF-8 ${ }^{\mathbf{2 0 , 3 9}}$ but slightly smaller than the values known for bulk ZIF-8 $\left(\sim 2000 \mathrm{~m}^{2} \mathrm{~g}^{-1}\right) .{ }^{40}$ It is due to the ratio of the external surface and the internal pore area increases with the decreasing size of a porous crystal into the nano-range, resulting in the loss of surface area. ${ }^{16}$ As a general trend, the surface area of MOFs is known to slightly decrease with decreasing size of the porous crystals especially when the crystal is at nanoscale, which is the opposite trend of surface area for non-porous nanoparticles. In this study, we only focus on nanosized ZIF-8 since large ZIF-8 crystals have been reported previously for removal of organic dyes. ${ }^{7,15,16}$

\subsection{Kinetics studies of RhB adsorption by ZIF-8}

The absorbance of RhB in the mixture solution with ZIF-8 was monitored by a spectrometer at $554 \mathrm{~nm}$. The absorbance was normalized to the starting RhB solution without the presence of ZIF-8 nanoparticles. The concentration of RhB decreases with the increasing stirring time with ZIF-8 nanoparticles in dark, visible light, and UV conditions, as shown in Fig. 3. The absorbance decreased more dramatically when the sample was exposed to visible light and UV irradiation compared to the complete dark environment. This indicates ZIF-8 is a better adsorbent for RhB with light. In all three cases, the initial $\mathrm{pH}$ remained the same at $\sim 8.0$, the slightly basic $\mathrm{pH}$ was a result of the basicity of ZIF- $8,{ }^{8,41}$ which is favorable for adsorbing cationic dyes like RhB. The concentration of RhB in solution decreased significantly within the first 10 minutes, indicating fast RhB adsorption by ZIF-8 nanocrystals even in dark. Adsorption

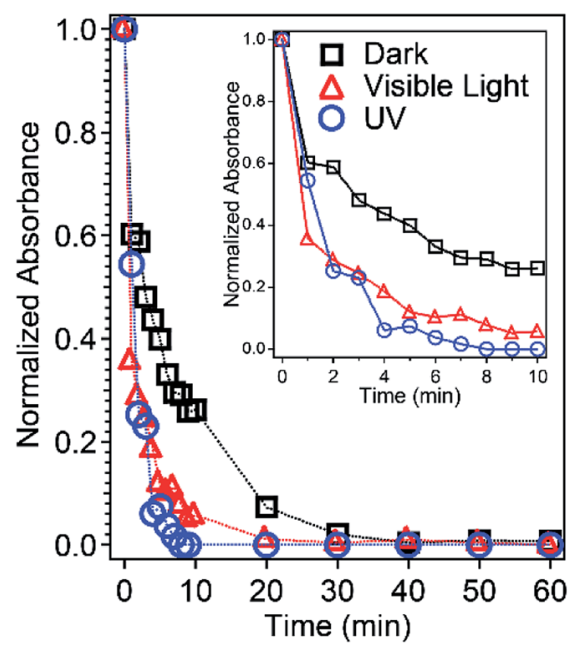

Fig. 3 Normalized UV-Vis absorbance of RhB in water with ZIF-8 under dark (black), visible light (red), and UV (blue) conditions. The inset is an expansion of the first $10 \mathrm{~min}$. equilibrium was reached within $60 \mathrm{~min}$ for all three test conditions. The self-degradation of RhB was negligible under dark and visible light up to 24 hours and under UV irradiation for 1 hour, as indicated by a slight decrease (1\%) in absorbance shown in Fig. S2. $\dagger$ Thus, it confirms the RhB degradation was mainly due to the presence of ZIF-8 nanocrystals in the mixture solution. The stability of ZIF-8 in water was also studied by taking PXRD of ZIF-8 before and after being immersed in water for the tested period. No noticeable changes were found on the PXRD patterns (Fig. S6†), indicating ZIF-8 nanocrystals did not decompose in water even under UV irradiation for at least 1 hour. The self-degradation control experiments of RhB and ZIF8 in water are summarized in the ESI. $\dagger$

The adsorption capacity $\left(q_{\mathrm{t}}\right)$ of ZIF-8 toward RhB was estimated based on the following equation:

$$
q_{t}=\left(C_{0}-C_{t}\right) \times V / m
$$

where $C_{0}$ is the initial $\mathrm{RhB}$ concentration, $C_{t}$ is the $\mathrm{RhB}$ concentration at time $t, V$ is the total volume of sample solution and $m$ is the mass of ZIF- 8 applied in the mixture solution. The adsorption kinetics of RhB was explored based on two models: pseudo-first-order and pseudo-second-order. The kinetics rate constants were calculated, respectively.

3.2.1 Pseudo-first-order model. The pseudo-first-order model is based on the Lagergren equation which has been widely used for describing adsorption kinetics of organics in aqueous solutions. ${ }^{42}$ Recent studies show methylene blue adsorption by selective MOFs, such as ZIF-8 and MIL-53(Fe), have followed this model. ${ }^{7,43}$ The pseudo-first-order equation can be expressed as follows:

$$
\log \left(q_{\mathrm{m}}-q_{t}\right)=\log \left(q_{\mathrm{m}}\right)-\frac{k_{1} t}{2.303}
$$

where $q_{t}$ and $q_{\mathrm{m}}$ are the adsorbed amounts $\left(\mathrm{mg} \mathrm{g}^{-1}\right)$ at time $t$ ( $\mathrm{min})$ and at equilibrium, $k_{1}\left(\mathrm{~min}^{-1}\right)$ is the corresponding adsorption rate constant. Based on the kinetics data shown in Fig. 3, an equilibrium was reached for the RhB/ZIF-8 system at all three conditions within $30 \mathrm{~min}$. Herein, we assume the adsorbed amount at the maximum contact time (60 $\mathrm{min}$ ) as the $q_{\mathrm{m}}$ value. Fig. 4 a shows the plots of $\log \left(q_{\mathrm{m}}-q_{t}\right)$ versus $t$ which are used to validate the applicability of the pseudo-first-order model. The calculated adsorption rate constants and correlation coefficients are summarized in Table 1 . Since the equilibria were achieved within the testing period, making some of $\log \left(q_{\mathrm{m}}\right.$ $-q_{t}$ ) values invalid, thus only non-zero values were considered in this model. For many adsorption systems, the pseudo-firstorder kinetics model is found to be suitable for a limited period of time at the initial contact stage and not for the whole range of adsorption process. ${ }^{\mathbf{4 4 , 4 5}}$

3.2.2 Pseudo-second-order model. We also applied a pseudo-second-order model to study the adsorption kinetics of RhB on ZIF-8 nanoparticles. Several studies have shown adsorption of organic molecules over ZIF-8 follows the pseudosecond-order kinetics behavior. ${ }^{46,47}$ The pseudo-second-order model, as shown below, has been applied broadly for solute adsorption and catalysis reactions in liquid conditions: ${ }^{48}$ 

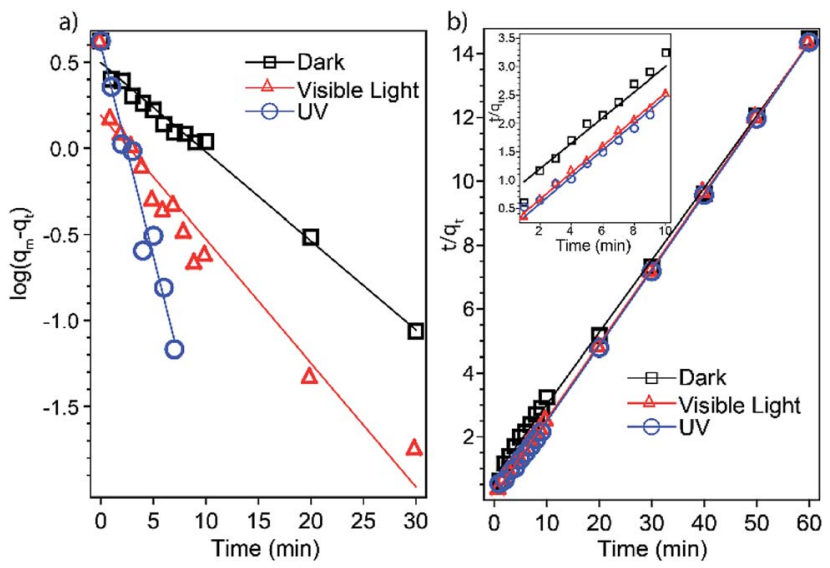

Fig. 4 Adsorption kinetics based on (a) the pseudo-first-order model and (b) the pseudo-second-order model. The inset is an expansion of the first $10 \mathrm{~min}$

$$
\frac{t}{q_{t}}=\frac{1}{k_{2} q_{\mathrm{e}}^{2}}+\frac{t}{q_{\mathrm{e}}}
$$

where $q_{t}$ and $q_{\mathrm{e}}$ are the adsorbed amounts $\left(\mathrm{mg} \mathrm{g}^{-1}\right)$ at time $t$ (min) and at equilibrium, $k_{2}\left(\mathrm{~g} \mathrm{mg}^{-1} \mathrm{~min}^{-1}\right)$ is the corresponding rate constant. The higher correlation coefficients were observed in the pseudo-second-order model for all three conditions, shown in Fig. $4 \mathrm{~b}$ and Table 1, indicating the RhB adsorption over ZIF-8 nanocrystals fits better to the pseudosecond-order adsorption kinetics. The highest kinetics rate constant in the UV condition is almost 10 times greater than that in dark. It indicates the adsorption of RhB by ZIF-8 is more efficiently under UV irradiation. The adsorption mechanisms will be discussed in the next part.

\subsection{Adsorption mechanism studies}

3.3.1 DFT computational studies of possible interactions between RhB and ZIF-8. Since different adsorption rates of RhB on ZIF-8 were observed, it is worth investigating the adsorption/ degradation mechanisms. We started by exploring the electronic properties of each material via cluster DFT simulations. Fig. 5 compares the highest occupied molecular orbital (HOMO) of RhB and the lowest unoccupied molecular orbitals (LUMOs) of ZIF-8 with $\mathrm{Zn}$ ions in various coordination environments and with multifunctional groups. Chizallet and co-workers studied the external surface groups of ZIF-8 by using a cluster approach and confirmed the accuracy of the model clusters compared to the periodic structure of ZIF- $8 .^{24,49}$ Inspired by their work, we

Table 1 Kinetics constants and correlation coefficients

\begin{tabular}{llllll}
\hline & \multicolumn{2}{l}{ Pseudo-first-order } & & \multicolumn{2}{l}{ Pseudo-second-order } \\
\cline { 2 - 3 } Condition & $k_{1}\left(\mathrm{~min}^{-1}\right)$ & $R^{2}$ & & $k_{2}\left(\mathrm{~g} \mathrm{mg}^{-1} \mathrm{~min}^{-1}\right)$ & $R^{2}$ \\
\hline Dark & 0.1190 & 0.9877 & 0.0685 & 0.9986 \\
Ambient light & 0.1658 & 0.9271 & 0.3000 & 0.9999 \\
UV & 0.5631 & 0.9662 & 0.5027 & 0.9997
\end{tabular}

performed a full natural bond orbitals (NBO) analysis on ZIF-8 by employing seven models (Fig. 5). Among all, four models including $\left\{\mathrm{Zn}(\mathrm{mIm})_{4}\right\}^{2-}, \quad\left\{\mathrm{Zn}(\mathrm{mIm})_{3}\right\}^{-}, \quad\left\{\mathrm{Zn}(\mathrm{mIm})_{2}\right\}^{0}, \quad$ and $\{\mathrm{Zn}(\mathrm{mIm})\}^{+}$are used to represent ZIF-8 with center zinc ion connected by mIm ligands, while the low coordination number models (with 3, 2, and $1 \mathrm{mIm}$ ligand) are considered as the structures with open metal defects (Fig. 5c-e). In the frontier orbital analysis, the HOMO of RhB is delocalized over the xanthene ring; the LUMOs of ZIF-8 (Fig. 5b-e) are primarily localized on the zinc ion, making the low coordinated $\mathrm{Zn}$ ions as Lewis acid sites. Thus, in principle, it is expected that nitrogen atoms and the xanthene ring of $\mathrm{RhB}$ will interact with the open metal sites of ZIF-8. With fewer methylimidazole ligands, the rendered isosurface becomes larger, indicating the LUMO of uncoordinated Zn-containing ZIF-8 has a greater chance to be approached and attacked by RhB molecules. Due to the steric hindrance effect, it is difficult to form covalent bonds between $\mathrm{RhB}$ and tetrahedrally coordinated ZIF-8. The open Zn sites provide not only orbitals for electron transfer, but also space for RhB molecules approaching to the unoccupied metal sites.

We also investigated the LUMOs of ZIF-8 with a variety of surface groups, including hydrogenocarbonate $\left(\mathrm{CO}_{3} \mathrm{H}-\right)$, hydroxide $(-\mathrm{OH})$, as well as secondary amine $(-\mathrm{NH}-)$. The three models (Fig. $5 \mathrm{f}-\mathrm{g}$ ) are created based on our previous experimental studies. ${ }^{21}$ We replaced one mIm ligand on ZIF-8 with the targeting surface functionality with a desired charge, except for the model of $\mathrm{NH}$ surface group (Fig. $5 \mathrm{~h})$ which is a $\mathrm{Zn}-(\mathrm{mIm})_{4}$ model with one imidazole modified with an -NH- group. We think the surface secondary amine are mainly generated by uncoordinated imidazole. The LUMO of the carbonate-terminated model (Fig. 5f) is delocalized on one of the imidazole rings, which may explain the $\pi-\pi$ stacking interaction adsorption mechanism. ${ }^{50}$ From the frontier orbital analysis, it is clear that the hydroxide-terminated ZIF-8 model (Fig. 5g) has the LUMO predominantly centered on the hydroxyl group, thus resulting in basic properties. Particularly, the hydroxyl groups may become one of the dominant surface groups of ZIF-8 when dissolved in water since more -OH terminations can be formed by water reacting with the low-coordinated zinc sites. The NH surface site (Fig. 5h) does not show any major impact on changing the LUMO of ZIF-8, but based on previous studies, the $\mathrm{NH}$ groups can act as Brønsted acid sites to donate protons to adsorbates. ${ }^{24}$ Overall, our frontier orbital analysis indicates the RhB interaction with ZIF-8 is likely a combined result of both metal open defects and surface groups. In order to verify the interaction process, experimental studies are followed.

3.3.2 Experimental studies of interactions between $\mathrm{RhB}$ and ZIF-8. Before proceeding to investigate the adsorption mechanism for RhB and ZIF-8 nanocrystals, the stability of ZIF8 toward RhB adsorption in water was addressed. We performed the aqueous stability test of ZIF-8 by taking PXRD of it before and after being soaked in water for 1 hour under UV exposure. We did not observe any changes on the PXRD patterns (Fig. S6 $\dagger$ ), indicating the stability of nanoscale ZIF-8 in water. However, we noticed an additional peak showing up at $11^{\circ}$ of $2 \theta$ angle in the PXRD interference pattern of ZIF-8 after mixing with RhB (Fig. 1a). We suspected this additional peak was related to the adsorbed $\mathrm{RhB}$ on ZIF-8 nanocrystals with 


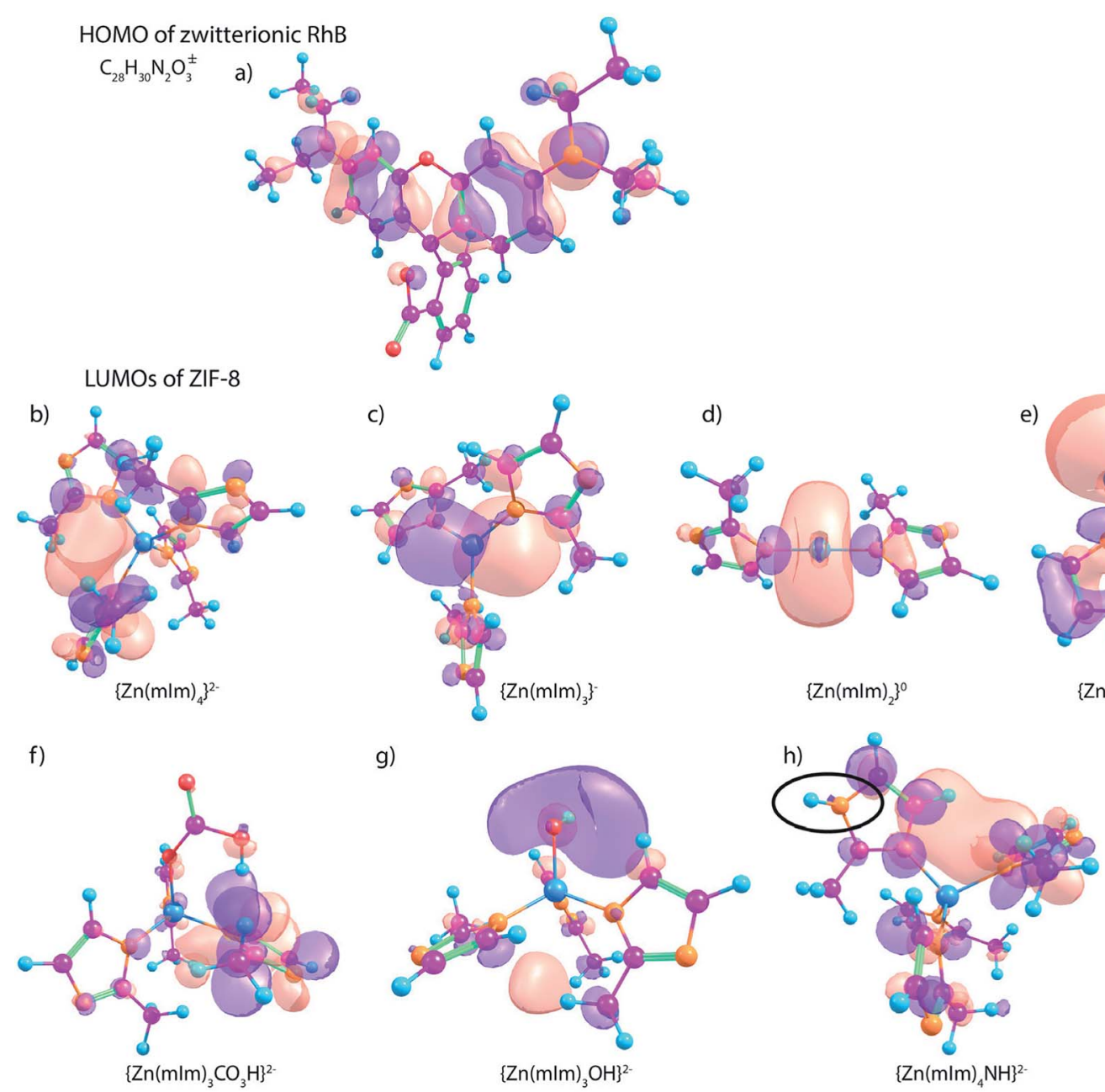

Fig. 5 DFT computationally predicted HOMO of the zwitterionic RhB ion (a) and LUMOs of ZIF-8 (b-h). ZIF-8 is represented by a cluster of $\left\{\mathrm{Zn}(\mathrm{mlm})_{x}\right\}^{(2-x)}$ with $x$ is an integer from 4 to 1 (model (b) to (e)). Model (f) and (g) refer to the ZIF- 8 external surface groups of hydrogenocarbonate and hydroxide, respectively. The surface secondary amine group is represented by model (h) with an $-\mathrm{NH}-\mathrm{moiety}$ on one mlm ligand, highlighted in a black circle. Carbon (purple), hydrogen (blue), nitrogen (orange), zinc (azure), and oxygen (red); positive and negative lobes are presented by orange and purple color with an isosurface value of 0.03 .

a preferred orientation, since extra interference patterns can be generated from the surface adsorbates when the testing material holds a significant surface-to-volume ratio. ${ }^{51,52}$ To further study this extra feature, we performed TEM measurements of ZIF-8 particles after RhB adsorption. We noticed some dark shaded rectangular-shaped crystals formed after the adsorption process (Fig. S7†). We suspected these crystals in a different shape may be due to the aggregation of RhB molecules adsorbed and accumulated on the outer surfaces of ZIF-8. We do not think the additional interference was caused by any guest molecule encapsulation because $\mathrm{RhB}$ has a relatively large molecular size with a kinetic diameter of $13.5 \AA$ i which cannot fit in the pore aperture of ZIF-8 (3.4 $⿱$ ). RhB molecules will aggregate and form zwitterionic dimers when the solution $\mathrm{pH}$ is greater than $4,{ }^{53}$ resulting in even larger size, further preventing the insertion of RhB into ZIF-8. Although several studies have confirmed the flexibility of ZIF-8 structure, ${ }^{54,55}$ its framework can only expand to certain degrees. Therefore, RhB molecules cannot enter ZIF-8 pores, which was also confirmed by our $\mathrm{N}_{2}$ sorption isotherms, as shown in Fig. 2. The amount of nitrogen adsorbed by ZIF-8 did not decrease after the RhB adsorption process, indicating the pores of ZIF-8 remained open. The increase in $\mathrm{N}_{2}$ adsorption amount after adsorbing RhB can be a result of the formation of surface adsorbates, as shown by the dark rectangular crystals observed in the TEM images (Fig. S7 $\dagger$ ). We suspected these surface adsorbates can generate excess external surface area. To quantify the change in external surface area of ZIF-8 before and after adsorbing RhB, we performed a $T$ plot study (Fig. S8 $\dagger$ ) and found that the external surface area was increased to $151 \mathrm{~cm}^{3} \mathrm{~g}^{-1}$, compared to that of pristine ZIF-8 nanocrystals of $113 \mathrm{~cm}^{3} \mathrm{~g}^{-1}$. The $T$-plot study is consistent with our TEM observation that extra crystals were formed among ZIF-8 nanoparticles. To explain the different kinetic behaviors of ZIF-8 toward RhB adsorption under dark and photon radiation conditions, we studied the following adsorption/degradation mechanisms.

3.3.2.1 Surface adsorption. As illustrated in our DFT cluster calculations, the defect sites and surface groups can interact 
with RhB molecules through Lewis acid sites and aromatic ring stacking effects. Additionally, these surface groups together with imidazole ligands make ZIF-8 particles negatively charged in aqueous, resulting in favorable adsorption for cationic RhB due to the electrostatic interaction.

To further study the surface interactions between RhB and ZIF-8, we performed XPS analysis on ZIF-8 nanocrystals before and after RhB adsorption. XPS is a surface analytical technique sensitive to the top $\sim 10 \mathrm{~nm}$ region; the penetration depth depends on the analyte material and the take-off angle of X-ray source. ${ }^{56}$ The high resolution spectra of $\mathrm{N}$ 1s and $\mathrm{Zn} 2 \mathrm{p}$ regions were broadened due to the RhB adsorption as shown in Fig. 6 . The $\mathrm{N}$ 1s peak at $398.3 \mathrm{eV}$ was corresponding to 2-methylimidazole in ZIF-8 based on the comparison to previously reported value for bulk ZIF-8. ${ }^{57}$ The $\mathrm{N}$ 1s peak at $400.4 \mathrm{eV}$ was assigned to the nitrogen feature from RhB. ${ }^{58}$ Our DFT calculations based on the Koopman's theorem ${ }^{30}$ confirmed that the $\mathrm{N}$ atoms from RhB have a higher core-level energy (399.66 eV) compared to the nitrogen in methylimidazole of ZIF-8 (397.61 $\mathrm{eV}$ ). The additional $\mathrm{N}$ 1s peak was a clear evidence of adsorbing RhB on the surface of ZIF-8. The surface hydroxide and carbonate groups on ZIF-8 would likely to attract quaternary ammonium cation in $\mathrm{RhB}$. In the $\mathrm{Zn} 2 \mathrm{p}$ region, the peaks at 1020.9 and $1043.9 \mathrm{eV}$ are corresponding to the $\mathrm{Zn}$ features of ZIF-8, including tetrahedrally coordinated $\mathrm{Zn}$ and zinc ions with low coordination numbers. ${ }^{21,57}$ After RhB adsorption, two additional peaks at 1022.6 and $1045.6 \mathrm{eV}$ show up, which are attributed to $\mathrm{RhB}$ attaching to the open $\mathrm{Zn}$ metal sites. XPS results along with TEM studies indicate $\mathrm{RhB}$ are adsorbed on ZIF-8 nanocrystals via surface groups but mainly through uncoordinated zinc defect sites, the two surface adsorption modes are illustrated in Scheme 1.

Because of the high surface-to-volume ratio of nanosized ZIF-8 compared to its bulk powder, more surface groups and defects are expected to be present. Therefore, ZIF-8 nanocrystals exhibit a faster and more effective adsorption toward RhB compared to its bulk material reported previously. ${ }^{16}$ To further
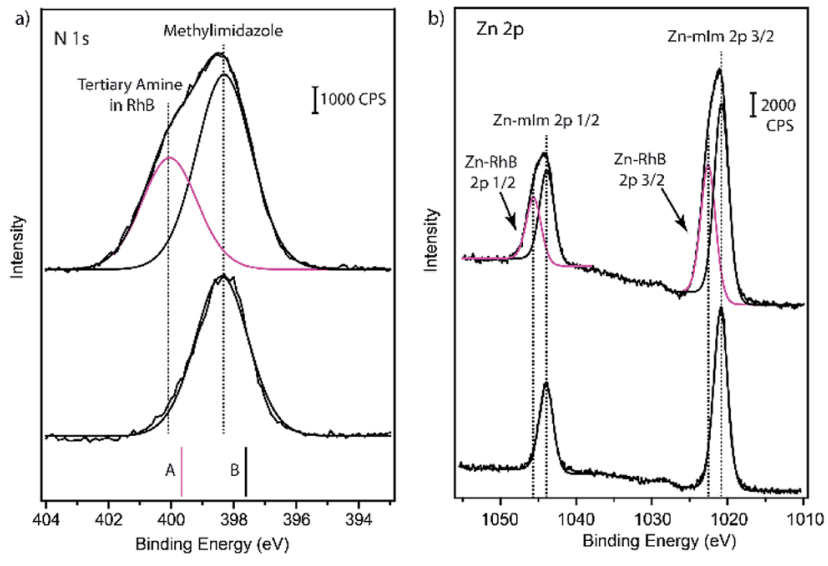

Fig. 6 High resolution (a) N 1s and (b) Zn 2p spectra of ZIF-8 nanoparticles before and after RhB adsorption ( 5 ppm under ambient light). Experimental $\mathrm{N}$ 1s spectra are compared with the DFT predicted corelevel energies (shown as solid bars) based on the computational structures $A$ and $B$ shown is Fig. 5.
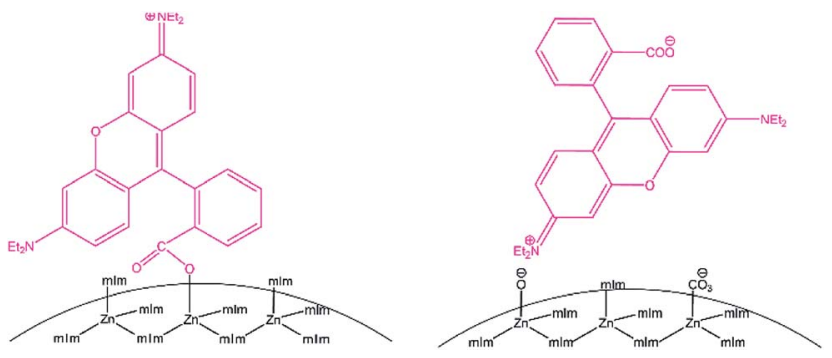

Scheme 1 Schematic illustration of surface adsorption modes for RhB on ZIF-8 nanoparticles based on uncoordinated Zn defects (left), and surface groups (right), such as hydroxide and carbonates.

understand the behaviors of ZIF- 8 and RhB after the initial solid-liquid adsorption process, the Weber and Morris intraparticle diffusion model ${ }^{59}$ was considered for all three adsorption conditions using the following equation:

$$
q_{t}=k_{\mathrm{id}} t^{1 / 2}+C_{\mathrm{i}}
$$

where $q_{t}$ is the adsorbed amount $\left(\mathrm{mg} \mathrm{g}^{-1}\right)$ at time $t(\mathrm{~min}), k_{\mathrm{id}}$ is the intraparticle diffusion coefficient, and $C_{\mathrm{i}}$ is a constant at stage $i$, relevant to the boundary effect on molecular diffusion at the liquid and solid interface. Based on the $q_{t}$ vs. $t^{1 / 2}$ plots shown in Fig. 7, the adsorption was confirmed to be separated into two stages: surface adsorption and intraparticle diffusion. The intraparticle model indicates RhB can be trapped in the space among ZIF-8 particles after the initial surface adsorption. The intraparticle diffusion model was also observed in previous $\mathrm{CO}_{2}$ and methanol experimental results. ${ }^{\mathbf{3 6 0}}$ Nevertheless the intraparticle diffusion did not promote the RhB molecules insertion into the ZIF-8 framework nor block the pore entrance pathway confirmed by our $\mathrm{N}_{2}$ sorption isotherm studies.

If surface adsorption is the only adsorption mechanism, we should not observe the different kinetics behaviors at various dark/light conditions. The adsorption is faster after exposure to visible light and UV irradiation; we attribute it to the photocatalytic degradation.

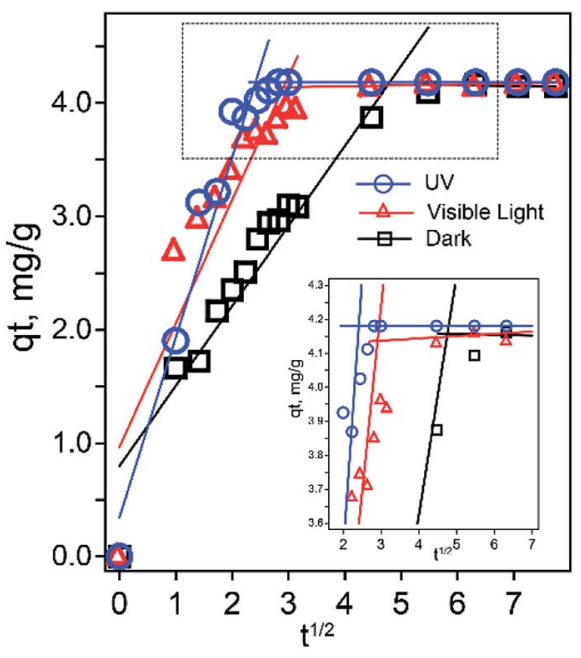

Fig. 7 Intraparticle diffusion of RhB into ZIF-8 nanoparticles under dark, ambient light, and UV conditions simulated using absorbance data. The inset is an expansion of the boxed area. 
3.3.2.2 Photocatalytic degradation. It has been well studied that organic dye molecules can be photodegraded by semiconductors through two pathways: (a) direct semiconductor excitation and (b) indirect dye sensitization. For example, Zhao et al., reported the photoassisted degradation of $\mathrm{RhB}$ by $\mathrm{TiO}_{2}$ particles under visible light irradiation through an indirect dye sensitization pathway, during which RhB molecules function as electron donors (eject electrons) while solid oxides are the electron-transfer mediators. ${ }^{61}$ Chandra et al., reported that methylene blue dye can be photodecomposed by hydroxyl radicals generated by $\mathrm{TiO}_{2} \mathrm{NPs} @ Z$ ZIF-8 core-shell heterostructures under UV-visible condition. ${ }^{62}$ To verify the photodegradation mechanism of RhB by nanosized ZIF-8. We first employed DFT cluster calculations to compare the electronic potentials of ZIF-8 with other inorganic solids, including $\mathrm{ZnO}$ and rutile $\mathrm{TiO}_{2}$, which have been studied extensively for photocatalytic degradation of RhB.

In our simulation studies, ZIF-8 exhibits a large HOMOLUMO energy gap ( $5.6 \mathrm{eV}$ ) which is comparable to the experimentally measured optical band gap $(4.9 \mathrm{eV})^{63}$ and the predicted band gap (5.5 eV) via periodic DFT calculations. ${ }^{64}$ Visible light can provide photon energy in a range of 1.8 to $3.9 \mathrm{eV}$; not enough to trigger the electron excitation in ZIF-8, as illustrated in Scheme 2. But under visible light irradiation, electrons can be excited within $\mathrm{RhB}\left(E_{\mathrm{g}}=2.9 \mathrm{eV}\right)$ and generate singlet and triplet excited states $\left(\mathrm{RhB}^{*}\right)$. Previous studies confirmed $\mathrm{RhB}^{*}$ can inject electrons into the closer conduction band of the semiconductor adsorbent (e.g. $\mathrm{TiO}_{2}$ ), meanwhile $\mathrm{RhB}$ will be converted to radical cations. ${ }^{\mathbf{6 1}}$ After accepting the injected electrons, $\mathrm{TiO}_{2}$ reacts with surface oxidants and produces oxygen radicals, such as $\mathrm{O}_{2}{ }^{--},{ }^{\circ} \mathrm{OOH}$, and ${ }^{\circ} \mathrm{OH}$. In turn, these highly reactive oxygen species (ROS) will react with surface

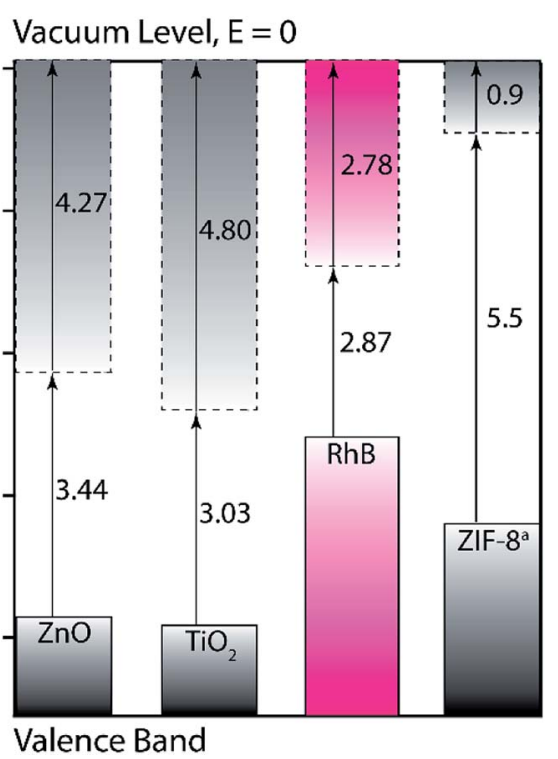

Scheme 2 Comparison of calculated electronic energy levels of RhB, $\mathrm{ZIF}-8, \mathrm{ZnO}$, and rutile $\mathrm{TiO}_{2}$ (in eV respect to vacuum level). Dash and solid boxes represent conduction band and valence band, respectively. ${ }^{a} V$ alues were taken from DFT calculations with periodic boundary conditions. ${ }^{64}$ The values for $\mathrm{ZnO}$ and $\mathrm{TiO}_{2}$ are taken from previously reported DFT predicted bandgap. ${ }^{70,71}$ adsorbed RhB radical cations and form degradation products. ${ }^{61}$ This describes the mechanism of the indirect dye sensitization. However, in the case of RhB adsorption on ZIF-8, the electron transfer from RhB to ZIF-8 is restricted due to the higher conduction band of ZIF-8 in comparison to that of RhB, further limiting the generation of oxygen radicals. In fact, there was no hydroxyl radicals observed by the terephthalic ${ }^{\circ} \mathrm{OH}$ probe using fluorescence spectroscopy (Fig. S4 $\dagger$ ). The visible photo-induced degradation of $\mathrm{RhB}$ is due to the enhanced surface adsorption by ZIF-8 nanocrystals. After exposure to visible light irradiation, valence electrons can be excited within rhodamine B molecules and form the excited states. These excited rhodamine B molecules can have higher energy and stronger interactions with the surface groups of ZIF-8, resulting in a more favorable and faster surface adsorption. We think the faster adsorption of RhB by ZIF-8 in visible light is due to the enhanced interactions between reactive $\mathrm{RhB}^{*}$ and surface groups of ZIF-8, since excited $\mathrm{RhB}^{*}$ are more efficiently being adsorbed on the surface and form $\mathrm{RhB}_{\mathrm{ads}}^{*}{ }^{\mathbf{6 1 , 6 5}}$

In our kinetics studies, we noticed the UV radiation resulted in the fastest adsorption rate. We hypothesized it was a result of $\mathrm{RhB}$ degradation by $\mathrm{OH}$ radicals generated from ZIF-8 through a direct excitation pathway. ${ }^{\mathbf{6}}$ Under UV irradiation, valence electrons of ZIF-8 are excited to the conduction band, followed by reacting with surface oxygen species and form oxygen radicals, as summarized in Scheme 3. Our study confirmed nanosized ZIF-8 are able to generate hydroxyl radicals. We used terephthalic acid as an ${ }^{\circ} \mathrm{OH}$ probe in $\mathrm{NaOH}$ solution. Terephthalic acid can react with hydroxyl radicals and form 2-hydroxyterephthalic acid which emits fluorescence at $430 \mathrm{~nm} \cdot{ }^{67}$ By monitoring the fluorescence intensity of the formed 2-hydroxyterephthalic acid, we are able to probe the amount of hydroxyl radicals generated by ZIF-8 nanocrystals. Fig. 8a shows the emission peak at $430 \mathrm{~nm}$ in the photoluminescence (PL) spectra of ZIF-8 solution and the peak intensity increases with increasing stirring time. Our results indicated ${ }^{\circ} \mathrm{OH}$ radicals can be produced by ZIF-8 only under UV irradiation but not in the visible light illumination. We also performed the control experiments with only terephthalic acid in $\mathrm{NaOH}$ solution under UV condition and no signal was observed at $430 \mathrm{~nm}$ up to 120 min test period (Fig. S4 $\dagger$ ). Our results confirmed that the 'OH radicals were generated by ZIF-8 nanocrystals after exposure to UV radiation. Highly reactive ${ }^{\circ} \mathrm{OH}$ radicals are well-
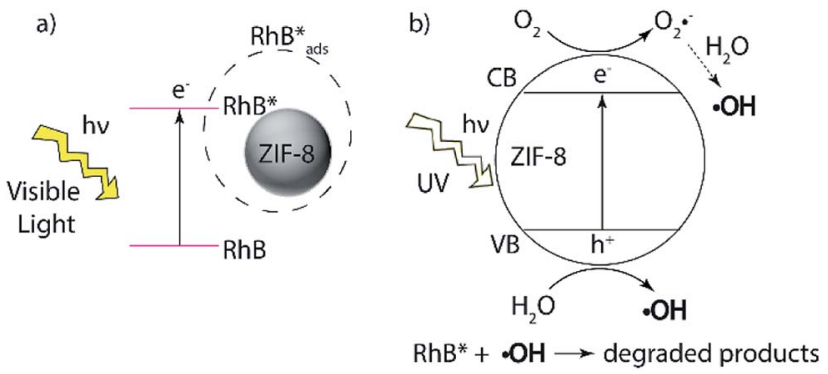

Scheme 3 Proposed mechanisms of photoassisted RhB adsorption/ degradation by ZIF-8 nanoparticles under (a) visible and (b) UV irradiation. 

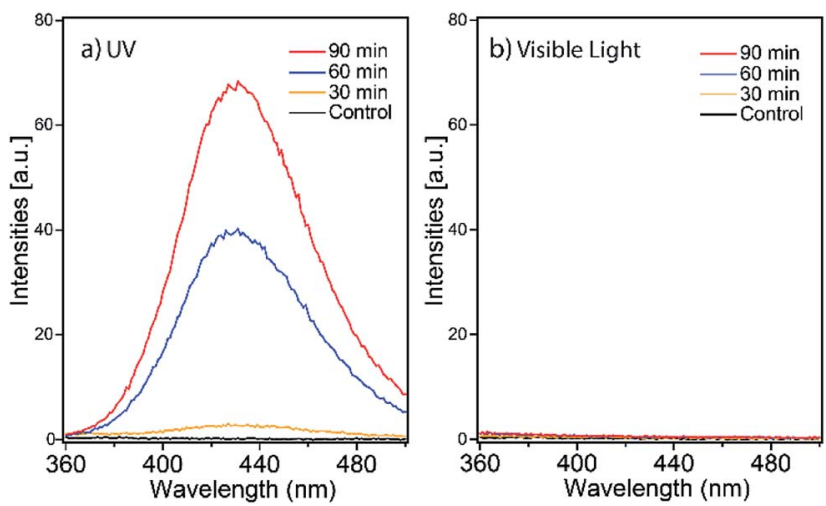

Fig. 8 Fluorescence spectra of terephthalic acid as an ${ }^{\circ} \mathrm{OH}$ radical probe in the presence of ZIF-8 under (a) UV and (b) visible light irradiation. The spectra were taken with an excitation wavelength of $315 \mathrm{~nm}$.

known to be able to react with organic dyes through the advanced oxidation processes (AOPs). ${ }^{68}$ The proposed mechanism of RhB photodegraded by ZIF-8 nanoparticles is summarized in Scheme 3. The analysis of photo-induced degradation byproducts was attempted using GC-MS, however, due to the low concentration and strong polar interactions of the hydroxylated compounds in water, we could not obtain qualitative results to confirm the degradation intermediates. The photo degradation products of $\mathrm{RhB}$ by ${ }^{\circ} \mathrm{OH}$ radicals and possible reaction pathways have been reported previously. ${ }^{10,62,69}$ More importantly, we did not observe any evidence of photodegradation of ZIF-8 after the UV adsorption experiments as indicated by IR and XRD studies, suggesting the stability and regeneration capability of ZIF-8 (Fig. S5 $\dagger$ ). We believe the fastest adsorption of RhB by ZIF-8 nanoparticles in UV condition was due to all the factors: surface adsorption, visible light enhanced adsorption, and the ${ }^{\circ} \mathrm{OH}$ radicals produced by ZIF- 8 through direct excitation under UV radiation.

In addition, we performed similar adsorption experiments on other organic dye molecules, including methylene blue, fluorescein, and rhodamine $6 \mathrm{G}$ under visible light condition. We noticed nanosized ZIF-8 worked well on rhodamine 6G, about $80 \%$ absorption decrease after $1 \mathrm{~h}$ of immersion (Fig. S9†). We believe this is due to the surface interaction between ZIF-8 and cationic rhodamine 6G which has a similar molecular structure to RhB. More experimental work will be needed to study the details of the adsorption mechanisms. We will also explore the possibility of employing commercially available ZIF-8 (Basolite Z1200) in water remediation as our future work.

\section{Conclusions}

In summary, ZIF-8 nanoparticles were found to be capable of adsorbing organic dye RhB even in dark condition with a fast adsorption rate. This is due to the rich multifunctional surface groups and defects resulting from the high surface-to-volume ratio of nanosized ZIF-8. The surface groups and defects of ZIF-8 facilitate the RhB adsorption. A faster adsorption rate of
RhB over ZIF-8 was noticed under photo-illumination. After the studies of electronic band structures and hydroxyl radicals, we confirmed RhB was photodegraded by ZIF-8 nanoparticles through two different mechanisms: under visible light irradiation, the electron excitation takes place in $\mathrm{RhB}$ and result in an enhanced surface adsorption on ZIF-8. While under UV irradiation, ZIF-8 itself is excited and produces electrons and holes which further react with surface oxidants and generate highly reactive hydroxyl radicals for photoassisted degradation of RhB. Additionally, we experimentally identified that RhB adsorption by ZIF-8 followed a pseudo-second-order model for all three conditions. Overall, our combined experimental and computational studies have provided a mechanistic insight on RhB adsorption/degradation on nanosized ZIF-8. We hope this work can shed light on organic molecular adsorption by other nanoMOFs or on the incorporation of nano-MOFs in membranes for photocatalytic reactions.

\section{Conflicts of interest}

There are no conflicts to declare.

\section{Acknowledgements}

We acknowledge funding from the Environmental Research and Education Foundation, as well the financial support provided by the California State University Long Beach and the John and Elizabeth Leonard Family Foundation. Research reported in this publication was partially supported by the National Institute of General Medical Sciences of the National Institutes of Health under Award Number T34GM008074. The content is solely the responsibility of the authors and does not necessarily represent the official views of the National Institutes of Health. The authors would like to acknowledge Dr Shahab Derakhshan and Andy Sua (CSULB) for XRD support, Dr Ich Tran (University of California, Irvine) for XPS support, and Dr Varenka Lorenzi for GC-MS support. FT is grateful for the helpful discussions with Drs Katarzyna Slowinska and Xianhui $\mathrm{Bu}$ (CSULB).

\section{Notes and references}

1 C. A. Martínez-Huitle and E. Brillas, Appl. Catal., B, 2009, 87, 105-145.

2 A. Y. Zahrim, C. Tizaoui and N. Hilal, Desalination, 2011, 266, 1-16.

3 B. Van Der Bruggen, C. Vandecasteele, T. Van Gestel, W. Doyen and R. Leysen, Environ. Prog., 2003, 22, 46-56.

4 V. K. Gupta and Suhas, J. Environ. Manage., 2009, 90, 23132342.

5 H. Furukawa, K. E. Cordova, M. O'Keeffe and O. M. Yaghi, Science, 2013, 341, 974-987.

6 C. Wang, X. Liu, J. P. Chen and K. Li, Sci. Rep., 2015, 5, 16613.

7 H.-P. Jing, C.-C. Wang, Y.-W. Zhang, P. Wang and R. Li, RSC Adv., 2014, 4, 54454-54462.

8 J. Li, Y. Wu, Z. Li, B. Zhang, M. Zhu, X. Hu, Y. Zhang and F. Li, J. Phys. Chem. C, 2014, 118, 27382-27387. 
9 H. Zhang, J. Hou, Y. Hu, P. Wang, R. Ou, L. Jiang, J. Z. Liu, B. D. Freeman, A. J. Hill and H. Wang, Sci. Adv., 2018, 4, eaaq0066.

10 E. M. Dias and C. Petit, J. Mater. Chem. A, 2015, 3, 2248422506

11 Z. Hasan and S. H. Jhung, J. Hazard. Mater., 2015, 283, 329339.

12 S. Bai, L. Wang, Z. Li and Y. Xiong, Adv. Sci., 2017, 4, 1600216.

13 Z. Wang, J. Liu, H. K. Arslan, S. Grosjean, T. Hagendorn, H. Gliemann, S. Bräse and C. Wöll, Langmuir, 2013, 29, 15958-15964.

14 M. T. Luebbers, T. Wu, L. Shen and R. I. Masel, Langmuir, 2010, 26, 15625-15633.

15 T.-T. Han, J. Yang, Y.-Y. Liu and J.-F. Ma, Microporous Mesoporous Mater., 2016, 228, 275-288.

16 X. Fan, W. Wang, W. Li, J. Zhou, B. Wang, J. Zheng and X. Li, ACS Appl. Mater. Interfaces, 2014, 6, 14994-14999.

17 X. Zhang, Y. Liu, S. Li, L. Kong, H. Liu, Y. Li, W. Han, K. L. Yeung, W. Zhu, W. Yang and J. Qiu, Chem. Mater., 2014, 26, 1975-1981.

18 C. H. Kuo, Y. Tang, L. Y. Chou, B. T. Sneed, C. N. Brodsky, Z. Zhao and C. K. Tsung, J. Am. Chem. Soc., 2012, 134, 14345-14348.

19 A. J. Howarth, Y. Liu, P. Li, Z. Li, T. C. Wang, J. T. Hupp and O. K. Farha, Nat. Rev. Mater., 2016, 1, 15018.

20 J. Cravillon, S. Münzer, S. J. Lohmeier, A. Feldhoff, K. Huber and M. Wiebcke, Chem. Mater., 2009, 21, 1410-1412.

21 F. Tian, A. M. Cerro, A. M. Mosier, H. K. Wayment-Steele, R. S. Shine, A. Park, E. R. Webster, L. E. Johnson, M. S. Johal and L. Benz, J. Phys. Chem. C, 2014, 118, 14449-14456.

22 C. Kropf, H. Segner and K. Fent, Comp. Biochem. Physiol., Part C: Toxicol. Pharmacol., 2016, 185-186, 45-56.

23 S. He, W. Xie, W. Zhang, L. Zhang, Y. Wang, X. Liu, Y. Liu and C. Du, Spectrochim. Acta, Part A, 2015, 137, 1092-1099.

24 C. Chizallet and N. Bats, J. Phys. Chem. Lett., 2010, 1, 349353.

25 P. J. Hay and W. R. Wadt, J. Chem. Phys., 1985, 82, 270-283.

26 W. R. Wadt and P. J. Hay, J. Chem. Phys., 1985, 82, 284-298.

27 P. J. Hay and W. R. Wadt, J. Chem. Phys., 1985, 82, 299-310.

28 J. M. L. Martin and A. Sundermann, J. Chem. Phys., 2001, 114, 3408-3420.

29 D. Feller, K. A. Peterson, W. A. de Jong and D. A. Dixon, J. Chem. Phys., 2003, 118, 3510-3522.

30 T. R. Leftwich and A. V. Teplyakov, J. Electron Spectrosc. Relat. Phenom., 2009, 175, 31-40.

31 S. R. Venna and M. a. Carreon, J. Am. Chem. Soc., 2010, 132, 76-78.

32 A. Demessence, C. Boissière, D. Grosso, P. Horcajada, C. Serre, G. Férey, G. J. a. a. Soler-Illia and C. Sanchez, J. Mater. Chem., 2010, 20, 7676.

33 G. Lu and J. T. Hupp, J. Am. Chem. Soc., 2010, 132, 78327833.

34 A. Galve, N. Liédana, A. Galve, C. Rubio, C. Téllez and J. Coronas, ACS Appl. Mater. Interfaces, 2012, 4, 5016-5021.

35 K. S. W. Sing, Pure Appl. Chem., 1985, 57, 603-619.
36 F. Tian, A. M. Mosier, A. Park, E. R. Webster, A. M. Cerro, R. S. Shine and L. Benz, J. Phys. Chem. C, 2015, 119, 15248-15253.

37 N. B. Nayak and B. B. Nayak, Sci. Rep., 2016, 6, 26404.

38 B. Naik, S. Hazra, V. S. Prasad and N. N. Ghosh, Catal. Commun., 2011, 12, 1104-1108.

39 J. Cravillon, C. a. Schröder, R. Nayuk, J. Gummel, K. Huber and M. Wiebcke, Angew. Chem., Int. Ed., 2011, 50, 8067-8071.

40 S. Gadipelli and Z. X. Guo, ChemSusChem, 2015, 8, 21232132.

41 I. U. Khan, M. H. D. Othman, A. F. Ismail, N. Ismail, J. Jaafar, H. Hashim, M. A. Rahman and A. Jilani, Mater. Charact., 2018, 136, 407-416.

42 H. Yuh-Shan, Scientometrics, 2004, 59, 171-177.

43 M. Hadi Mohd Tahir, S. Err Teo and P. Yan Moh, Transactions on Science and Technology, 2017, 4, 14-21.

44 K. V. Kumar, J. Hazard. Mater., 2006, 137, 1538-1544.

45 Y. C. Wong, Y. S. Szeto, W. H. Cheung and G. Mckay, J. Appl. Polym. Sci., 2004, 92, 1633-1645.

46 Y. Feng, Y. Li, M. Xu, S. Liu and J. Yao, $R S C A d v .$, 2016, 6, 109608-109612.

47 J.-Q. Jiang, C.-X. Yang and X.-P. Yan, ACS Appl. Mater. Interfaces, 2013, 5, 9837-9842.

48 Y.-S. Ho, J. Hazard. Mater., 2006, 136, 681-689.

49 C. Chizallet, S. Lazare, D. Bazer-Bachi, F. Bonnier, V. Lecocq, E. Soyer, A.-A. Quoineaud and N. Bats, J. Am. Chem. Soc., 2010, 132, 12365-12377.

50 K.-Y. A. Lin and H.-A. Chang, Water, Air, Soil Pollut., 2015, 226, 10.

51 R. M. Rioux, H. Song, J. D. Hoefelmeyer, P. Yang and G. A. Somorjai, J. Phys. Chem. B, 2005, 109, 2192-2202.

52 R. Felici, M. Pedio, F. Borgatti, S. Iannotta, M. Capozi, G. Ciullo and A. Stierle, Nat. Mater., 2005, 4, 688-692.

53 Q. Wang, C. Chen, D. Zhao, W. Ma and J. Zhao, Langmuir, 2008, 24, 7338-7345.

54 B. Zheng, Y. Pan, Z. Lai and K. W. Huang, Langmuir, 2013, 29, 8865-8872.

55 E. Haldoupis, T. Watanabe, S. Nair and D. S. Sholl, ChemPhysChem, 2012, 13, 3449-3452.

56 M. P. Seah and W. A. Dench, Surf. Interface Anal., 1979, 1, 211.

57 J. Yang, F. Zhang, H. Lu, X. Hong, H. Jiang, Y. Wu and Y. Li, Angew. Chem., 2015, 127, 11039-11043.

58 W. M. Liu, H. Y. Zhang, P. F. Wang, Q. Ye, Y. Yang, B. He, I. Bello, S. T. Lee and W. J. Zhang, Appl. Phys. Lett., 2011, 99, 063103.

59 W. Plazinski and W. Rudzinski, J. Phys. Chem. C, 2009, 113, 12495-12501.

60 A. M. Mosier, H. L. W. Larson, E. R. Webster, M. Ivos, F. Tian and L. Benz, Langmuir, 2016, 32, 2947-2954.

61 J. Zhao, T. Wu, K. Wu, K. Oikawa, H. Hidaka and N. Serpone, Environ. Sci. Technol., 1998, 32, 2394-2400.

62 R. Chandra and M. Nath, ChemistrySelect, 2017, 2, 77117722 .

63 F. Wang, Z.-S. Liu, H. Yang, Y.-X. Tan and J. Zhang, Angew. Chem., Int. Ed., 2011, 50, 450-453. 
64 K. T. Butler, C. H. Hendon and A. Walsh, J. Am. Chem. Soc., 2014, 136, 2703-2706.

65 F. Zhang, J. Zhao, L. Zang, T. Shen, H. Hidaka, E. Pelizzetti and N. Serpone, J. Mol. Catal. A: Chem., 1997, 120, 173-178.

66 D. Saliba, M. Ammar, M. Rammal, M. Al-Ghoul and M. Hmadeh, J. Am. Chem. Soc., 2018, 140, 1812-1823.

67 T. Hirakawa and Y. Nosaka, Langmuir, 2002, 18, 3247-3254.

68 O. Legrini, E. Oliveros and A. M. Braun, Chem. Rev., 1993, 93, 671-698.
69 Z. He, C. Sun, S. Yang, Y. Ding, H. He and Z. Wang, J. Hazard. Mater., 2009, 162, 1477-1486.

70 C. R. A. Catlow, S. A. French, A. A. Sokol, A. A. Al-Sunaidi and S. M. Woodley, J. Comput. Chem., 2008, 29, 2234-2249.

71 D. O. Scanlon, C. W. Dunnill, J. Buckeridge, S. A. Shevlin, A. J. Logsdail, S. M. Woodley, C. R. A. Catlow, M. J. Powell, R. G. Palgrave, I. P. Parkin, G. W. Watson, T. W. Keal, P. Sherwood, A. Walsh and A. A. Sokol, Nat. Mater., 2013, 12, 798-801. 